the duodenum, "neuromuscular and vascular hamartomas", and gangliocytic paragangliomas may be associated with many of the former lesions that are undoubtedly associated with mucosal damage such as that seen in Crohn's disease ${ }^{10}$ Furthermore, the occurrence of areas of neuromuscular hyperplasia, which may include ganglion cells, is well recognised in Crohn's disease. The histology of the tumours presented shows well circumscribed lesions with no adjacent mucosal abnormality. Case 1 seems to have arisen in the 12 month period between sigmoidoscopic examinations and may be directly related to the site of previous surgery. Although a mucosal lesion may seem to have healed, the chronic inflammatory reaction within the tumour may be evidence of previous surgery.

The identification of a gastrointestinal ganglioneuroma necessitates the clinical and histological consideration of further lesions or associated pathology. Indeed, in several patients ganglioneuromatous lesions of the gut have been the presenting features of multiple endocrine neoplasia syndrome type $2 b$.

Addendum

Since the preparation of this paper the occurrence of diffuse neuronal hyperplasia, in some cases including ganglion cells, has been highlighted in von Recklinghausen's disease by C E Fuller and G T Williams: Gastrointestinal manifestations of type 1 neurofibromatosis (von Recklinghausen's disease). Histopatholoy 1991;19:1-11.

I thank Mr B Sims for photographic assistance and Mrs P Bedevi and Mrs J A Daniel for typing the manuscript.
1 Poate $\mathrm{H}$, Inglis $\mathrm{K}$. Ganglioneuromatosis of the alimentary tract. Br J Surg 1928;16:221.
2 Hayes FA, Green AA, Rao BN. Clinical manifestations of ganglioneuromas. Cancer 1989;15:1211-14.
3 Carney JA, Go VLW, Sizemore GW, Hayles AB. Alimen- tary tract ganglioneuromatosis. A major component of the syndrome of M.E.N. type 2b. $N$ Engl J Med 1976; 295:1287-91.
4 Lashner BA, Riddell RH, Winans CS. Ganglioneuromatosis of the colon and extensive glycogenic acanthosis in Cowden's disease. Dig Dis Sci 1986;31:213.
5 Pham BN, Villanueva RP. Ganglioneuromatous prolifera- tion associated with juvenile polyposis coli. Arch Pathol Lab Med 1989;113:91-4.
6 Haff RC, San Diego AC. Ganglioneuroma of the ileocaecal valve. Review of the literature. Arch Pathol 1972;93:549.
7 Gemer M, Feuchtwanger MM. Ganglioneuroma of the duodenum. Gastroenterol 1966;51:689-93.
8 Sivak MV, Sullivan BH, Farmer RG. Neurogenic tumours of the small intestine. Review of the literature. Gastro- of the small intestine.
9 Daneshvar A. Pharyngeal traumatic neuromas and traumatic neuromas with mature ganglion cells (pseudoganglio- neuroma). Am J Surg Pathol 1990;14:565-70.
10 Shepherd NA, Jass JR. Neuromuscular and vascular hamartoma of the small intestine: is it Crohn's disease? Gut 1987;28:1663-8.

\title{
Rapid differentiation of Mycobacterium xenopi from mycobacteria of the Mycobacterium avium- intracellulare complex by pyrolysis mass spectrometry
}

\author{
P R Sisson, R Freeman, J G Magee, N F Lightfoot
}

Regional Public Health Laboratory, General Hospital, Westgate Road, Newcastle upon Tyne P R Sisson

J G Magee

N F Lightfoot

Medical School,

Framlington Place,

Newcastle upon Tyne

R Freeman

Correspondence to:

Dr R Freeman,

Microbiology Department,

Medical School

Framlington Place,

Newcastle upon Tyne

NE2 4HH.

Accepted for publication

5 September 1991

\begin{abstract}
Thirty four cultures of slow growing, Tween-80 negative mycobacteria were analysed by pyrolysis mass spectrometry. The results showed that pyrolysis mass spectrometry could positively distinguish strains of Mycobacterium xenopi from those of the Mycobacterium aviumintracellulare (MAI) complex. Pyrolysis mass spectrometry may be a useful technique for the rapid characterisation of non-tuberculous mycobacteria in such clinical settings as their isolation from immunocompromised example, those with AIDS.
\end{abstract}

Pyrolysis mass spectrometry is a rapid and simple technique for comparisons of strains of micro-organisms that has been successfully applied to a wide range of bacterial species. ${ }^{1}$ Pyrolysis mass spectra vary with the age and cultural conditions of the organisms before pyrolysis $^{2}$ so that pyrolysis mass spectrometry cannot assign permanent type designations. However, if organisms are prepared under identical conditions and then examined by pyrolysis mass spectrometry within a single machine batch, the system is highly discriminatory. Mycobacteria form a highly disparate group of organisms with a wide range of different growth cycles and optimal growth temperatures. Consequently, pyrolysis mass spectrometry is only likely to be useful when analysing within groups of mycobacteria of closely similar growth pattern. We recently adopted this approach in analysing members of the $M$ tuberculosis complex. Pyrolysis mass spectrometry was able to distinguish $M$ tuberculosis from $M$ bovis, and also showed the close 
similarity between $M$ bovis and $M$ africanum. ${ }^{3}$

An emerging clinical problem is the differentiation of mycobacteria isolated from immunocompromised patients, particularly those with AIDS. In most cases the organism is likely to belong to the Mycobacterium aviumintracellulare (MAI) complex, but recent reports suggest that other species such as $M$ xenopi can also infect these patients. ${ }^{45}$ An ability to distinguish rapidly $M$ xenopi from organisms of the MAI complex may become important in epidemiology and patient management.

Conventional differentiation relies on relative sensitivity to isoniazid, growth on tellurite containing media and thermophilicity. All these tests require subculture, take some time to complete, and are not always definitive. Detection of 2-Docosanol has been suggested as a rapid positive test for the identification of $M$ xenopi, but requires access to gas chromatography. ${ }^{6}$

On primary isolation both MAI complex and $M$ xenopi appear as slow-growing, Tween-80 negative mycobacteria. We have done a preliminary study to assess the potential of pyrolysis mass spectrometry to distinguish rapidly and positively $M$ xenopi from organisms of the MAI complex in this context.

\section{Methods}

Thirteen clinical isolates of $M$ xenopi (designated xen 1-xen 13), all of which had been characterised by conventional tests, were cultured in duplicate on Middlebrook $7 \mathrm{H} 10$ agar slopes (Difco Laboratories), together with a type strain of $M$ xenopi (NCTC 10042). All of the clinical isolates were obtained from different patients. Similar cultures, also in duplicate, were set up for one type strain (NCTC 8559), two reference strains (NCTC 8552 and 8562) of $M$ avium, and one type and one reference strain of $M$ intracellulare (NCTC 10682 and 10425). These cultures were incubated together with 15 clinical isolates of $M$ avium-intracellulare, six from AIDS sources and nine from non-AIDS sources (designated AI 1-AI 15), at $35^{\circ} \mathrm{C}$ for nine days, at which time a confluent growth was present on all the slopes.

Material from each subculture was smeared on to $\mathrm{Ni}-\mathrm{Fe}$ pyrolysis foils (Horizon Instruments Ltd, Heathfield, Sussex) in triplicate. The foils were inserted into pyrolysis tubes (Horizon Instruments) and heated at $80^{\circ} \mathrm{C}$ in a hot air oven for five minutes. The sample tubes were then processed on a Horizon Instruments PYMS 200X pyrolysis mass spectrometer. Each tube was pyrolysed for four seconds at a Curie point of $530^{\circ} \mathrm{C}$ and the ion counts were collected on to floppy disk and stored as previously described.

After normalisation of the ion counts, discriminant analysis was performed as previously described. ${ }^{7}$ The triplicate spectra from both subcultures of each isolate were labelled as distinct groups and analysed for intergroup
Figure Dendrogram showing percentage similarities between 14 isolates of Mycobacterium xenopi (13 clinical isolates and one type strain) and 20 isolates of the Mycobacterium aviumintracellulare complex (15 clinical isolates, three reference strains, and two type strains). The clustering method was UPGMA.

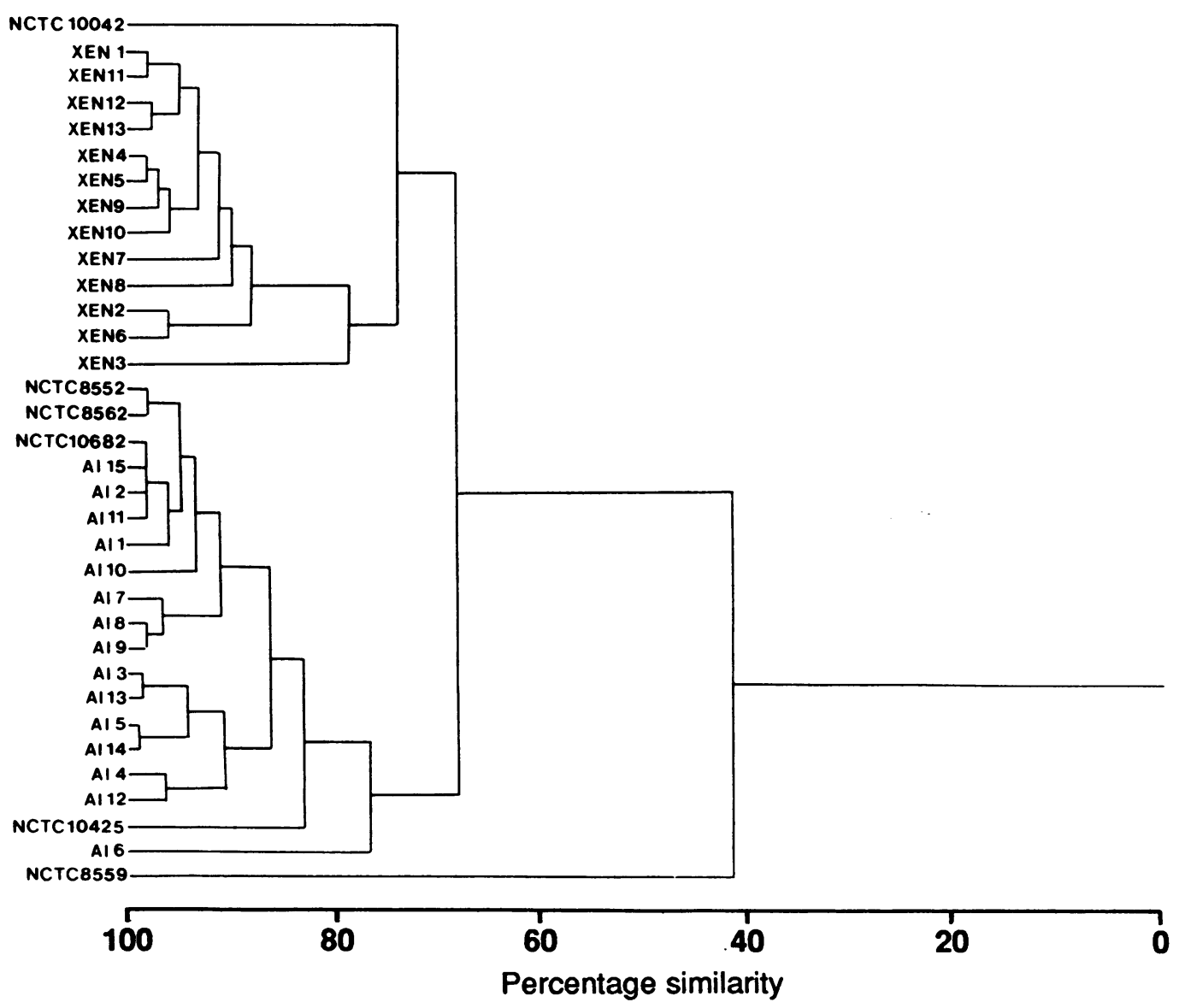


variation. Principal component and canonical variate analyses were then performed using the 30 mass ion peaks showing the greatest discrimination between groups, resulting in a table of Mahalanobis distances. These data were then used in a UPGMA analysis to produce a dendrogram.

\section{Results}

The dendrogram showed the relatedness of all 34 isolates of mycobacteria (figure). The 13 clinical isolates of $M$ xenopi closely resembled each other, 12 of the 13 clinical isolates showing $88 \%$ similarity, and all 13 being encompassed by $79 \%$ similarity. The most dissimilar isolate was the reference strain (NCTC 10042), which is included at $\mathbf{7 4 \%}$ similarity.

None the less all the $M$ xenopi isolates were clearly distinguished from the MAI clinical and reference isolates, these two groups only being related at $68 \%$ similarity. There was more heterogeneity within the MAI isolates than that seen within the $M$ xenop $i$ isolates, but all of the clinical isolates and reference strains were included within a $77 \%$ similarity.

Interestingly, the type strain of $M$ avium (NCTC 8559) was as different by pyrolysis mass spectrometry analysis from the wild and reference isolates of $\mathrm{MAI}$ as from the $M$ xenopi isolates. NCTC 8559 was linked to the whole of the remaining isolates, whether MAI or $M$ xenopi, at $41 \%$ similarity.

\section{Discussion}

This preliminary study suggests that pyrolysis mass spectrometry can clearly distinguish isolates of $M$ xenop $i$ from organisms of the MAI complex. The distinction is a positive one, the $M$ xenopi isolates being closely related one to another and distinct from the MAI isolates. In taxonomic terms $M$ xenop $i$ is seen to be a "good species". It is likely, therefore, that pyrolysis mass spectrometry analysis of a collection of slow growing, Tween-80 negative mycobacteria will permit a rapid positive characterisation of $M$ xenopi. Isolates excluded from the $M$ $x e n o p i$ cluster can be characterised as "not $M$ $x e n o p i$ " and therefore likely to belong to the MAI complex, but not positively identified as $M$ avium or $M$ intracellulare.

Pyrolysis mass spectrometry is a phenotypic comparative technique based on analysis of total cell content. Although results are valid and reproducible within machine batches, it is essential that the isolates to be examined are prepared under identical cultural conditions before pyrolysis. Such factors may have a bearing on the apparent discrepant result obtained with NCTC 8559, the type (not reference) strain of $M$ avium. Type strains are likely to differ in phenotype from fresh clinical isolates as a result of prolonged serial subculture. We noted a similar phenomenon in relation to the H37 RV strain of $M$ tuberculosis in a pyrolysis mass spectrometry study of the $M$ tuberculosis complex. ${ }^{3}$ When using pyrolysis mass spectrometry for rapid distinction between closely related groups of mycobacteria, it will be important to use both reference strains and well characterised recent clinical isolates in the comparison.

It is interesting to note the results of a recent study using capillary gas-liquid chromatography analysis of fatty acid, secondary alcohol, and mycolic acid content of mycobacteria in which characteristic results permitted direct positive identification of $M$ xenopi and $M$ malmoense; MAI complex organisms gave much more variable patterns. ${ }^{8}$ The differences in the pyrolysis mass spectrometry spectral data may be related to the presence or absence of these or similar compounds.

As only a few colonies of each organism are required for pyrolysis mass spectrometry analysis it can be performed on primary isolates of slow growing, Tween-80 negative mycobacteria, provided that appropriately grown cultures of $M$ avium, $M$ intracellulare, and $M$ xenopi are available for comparative purposes. Pyrolysis mass spectrometry is simple, relatively cheap, requires no special expertise in sample processing and provides definitive results on the same day.

We thank Dr PA Jenkins, Public Health Laboratory Service, Mycobacterium Reference Unit, for providing some of the isolates of Mycobacterium xenopi.

1 Pitt TL. Burnt offerings or typing system? J Hosp Infect 1991;17:157-8.

2 Voorhees KJ, Durfee SL, Updegraff DM. Identification of diverse bacteria grown under diverse conditions using pyrolysis-mass spectrometry. J Microbiol Methods 1988;
8.315-25. (315-25.

3 Sisson PR, Freeman R, Magee JG, Lightfoot NF. Differentiation between mycobacteria of the Mycobacterium tuberculosis complex by pyrolysis mass spectrometry Tubercle 1991;72:206-9.

4 Eng RHK, Forrester C, Smith SM, Sobel H. Mycobacterium xenopi infection in a patient with acquired immunodeficiency syndrome. Chest 1984;86:145-7.

5 Wilks D, George RJD, Spiro SJG. Pulmonary $M$ xenopi infection as a first manifestation of HIV infection. $J$ Roy Soc Med 1990;83:401-2.

6 Larsson L, Jiminez J, Valero-Guillen P, Martin-Luengo F, Kubin M. Establishment of 2-Docosanol as a cellular marker compound in the identification of Mycobacterium menopi. J Clin Microbiol 1989;27:2388-90.

7 Aries RE, Gutteridge CS, Ottley TW. Evaluation of a lowcost, automated pyrolysis mass spectrometer. J Anal Appl Pyrolysis 1986;9:81-98.

8 Luquin M, Ausina V, Calahorra Lopez F, et al. Evaluation of practical chromatographic procedures for identification of clinical isolates of mycobacteria. J Clin Microbiol 1991;29:120-30. 